International Journal of Wireless \& Mobile Networks (IJWMN) Vol. 6, No. 6, December 2014

\title{
Performance Evaluation Of Different Spectrum Sensing Techniques For Realistic IMPlementation ORIENTEd MOdel IN Cognitive RADIO NETWORKS
}

\author{
Dipak P.Patil ${ }^{1}$ and Vijay M.Wadhai ${ }^{2}$ \\ ${ }^{1}$ Department of Applied Electronics, SGBAU, Amravati, India \\ ${ }^{2}$ Sinhgad Academy of Engineering, Pune, India
}

\begin{abstract}
In this paper, the performance assessment of five different detection techniques from spectrum sensing perspective in cognitive radio networks is proposed and implemented using the realistic implementation oriented model (R-model) with signal processing operations. The performance assessment of the different sensing techniques in the existence of unknown or imprecisely known impulsive noise levels is done by considering the signal detection in cognitive radio networks under a non-parametric multisensory detection scenario. The examination focuses on performance comparison of basic spectrum sensing mechanisms as, energy detection (ED) and cyclostationary feature detection (CSFD) along with the eigenvalue-based detection methods namely, Maximum-minimum eigenvalue detection (MMED), Roy's largest Root Test (RLRT) which requires knowledge of the noise variance and Generalized Likelihood Ratio Test (GLRT) which can be implemented as a test of the largest eigenvalues vs. Maximum-likelihood estimates a noise variance. From simulation results it is observed that the detection performance of the GLRT method is better than the other techniques in realistic implementation oriented model.
\end{abstract}

\section{KEYWORDS}

Cognitive Radio, Cyclostationary feature detection, Energy detection, GLRT, RLRT, Spectrum Sensing

\section{INTRODUCTION}

The demand for the radio spectrum is dramatically increasing and is estimated to rise rapidly in the near future with reference to the rapid development of different wireless communication applications. The radio frequency spectrum is a limited natural resource to enable wireless communication between transmitters and receivers and it is already very packed. It appears that it is tough to accommodate more wireless applications within this limited resource. The frequency bands of the wireless communication spectrum are not currently used very efficiently, mainly due to the current static spectrum allocation policy is based on a fixed frequency allocation policy in which the licensed spectrum bands remains underutilized. The report of Federal Communications Commission (FCC) [1], exposes that in some locations or at some times of day, almost $70 \%$ of the allocated spectrum may be sitting idle. To deal with the discrepancy between spectrum congestion and spectrum under-utilization, cognitive radio (CR) has been recently proposed as the capable solution for improving the utilization of the available spectrum to current low usage of licensed spectrum problem [2]. A CR sense and identify spectrum opportunities unoccupied by a primary user (PU) and improve the spectrum utilization while reducing the white spaces in the spectrum. Also it must prevent interference with the licensed PUs. The spectrum sensing is one of the main issues from the spectrum management perspective as the CR needs to sense the spectrum holes in wireless environments before accessing the channel. [3]. 
International Journal of Wireless \& Mobile Networks (IJWMN) Vol. 6, No. 6, December 2014

Major problems for $\mathrm{CR}$ like multipath fading, receiver uncertainty hidden terminals and correlated shadowing observed in a non-cooperative spectrum sensing can be solved by cooperative spectrum sensing techniques. Cooperative sensing decreases the probabilities of missdetection and probability of false alarm considerably. Furthermore the hidden PU problem can also be solved which results in decrease in sensing time [4]. The basic spectrum sensing mechanisms are matched filter detection (MFD) [5-8], energy detection [9-12] and cyclostationary feature detection [13-15]. Every technique is unique in itself with certain advantages and limitations. The basic knowledge of cyclic frequencies of the primary signal is essential in CSFD, which may not be available to the secondary users in practice. Also, it has high computational complexity. MFD is considered to be an optimal signal-detection method. The MFD is assumed to be an optimal signal-detection method. However the prior knowledge of the PU such as, modulation type, pulse shaping, and synchronization of timing and carrier is indispensable. And in MFD, for each PU, the CR will require a committed receiver and this requirement is makes it difficult for practical implementation [16]. From [17- 19] it is observed that the eigenvalue-based spectrum sensing techniques is found as the best amongst existing sensing methods. The eigenvalue-based spectrum sensing methods has overcome the limitations of the previously discussed methods. The prior knowledge of the transmitted signal is not essential in this method. Also the most basic sensing method the ED is reasonably sensitive to the accuracy of the expected noise variance [20]. We have considered the effect of impulsive noise for investigating the performance of the eigenvalue- based spectrum sensing scheme in perspective of spectrum sensing.

Impulsive noise (IN) consists of repetitive or non-repetitive pulses with a random intensity, duration and occurrence. The major sources of IN generation are household appliances, heating systems, ignition devices and dropouts or surface degradation of audio recordings, clicks from computer keyboards [21-22]. Via selection and equal gain combining the performance of the ED is investigated and concluded that impulsive noise can degrade the sensing performance. The GLRT method of detection is also proposed. In the non-realistic conventional model no analysis has been made considering the different eigenvalue-based spectrum sensing methods.

\subsection{Conventional discrete time memory less linear MIMO fading channel model (C- Model)}

It is static model in which channel remains idle, we consider Additive White Gaussian Noise (AWGN) channel. The memory-less linear discrete-time multiple input multiple output (MIMO) fading channel for single-receiver, multi-sensor and multiple-receiver, single-sensor cognitive devices is considered. It is also known as conventional model (C-model) [23]. Because of limitation of no signal processing performed by the C-model, it cannot be used for multiple CR receivers, as the samples collected by each $\mathrm{CR}$ are considered and forwarded to the fusion centre directly. Hence modifications are essential in the $\mathrm{C}$-model.

\subsection{The Realistic Implementation oriented model (R-Model)}

The more realistic implementation- oriented MIMO (R-model), in which signal processing operations like filtering, quantization and automatic gain control (AGC) are used within directconversion CR receiver architecture as shown below [24-25]. 


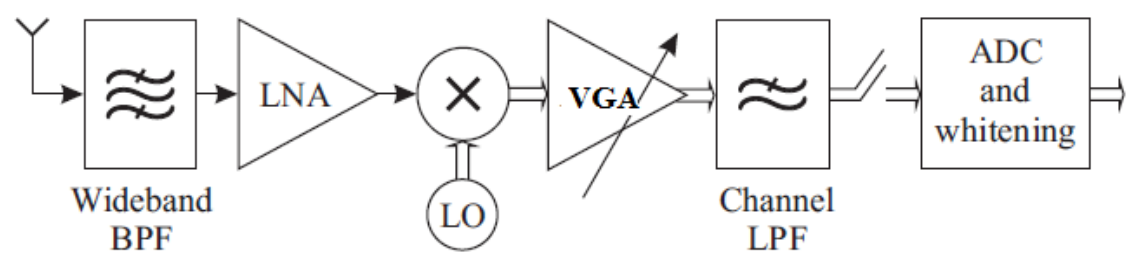

Figure 1. CR Receiver Diagram

Fig. 1 is considered for construction of the realistic model. In which the spectrum-sensing directed functions are combined with the direct conversion receiver (DCR). The wideband bandpass filter (BPF), low-noise amplifier (LNA) and quadrature local oscillators (LO) and mixers are used for direct conversion of the desired channel to in-phase and quadrature (I\&Q) baseband signals. The drawbacks like I\&Q imbalance are present in the DCRs, because it suffers from, flicker noise and DC- offset [26]. The DC-offset can effortlessly saturate succeeding amplification stages [27]. It is a DC signal appearing at the mixer output is composed of a static and a dynamic part, primarily due to LO self-mixing processes and in-band interfering signals. The static part can be removed by the careful circuit design and modern DC-offset compensation algorithms, but still some residual dynamic DC-offset will always remain [28]. The signal is held in reserve within the dynamic range of the analog-to-digital converters (ADC) in I\&Q signal paths which passed through the variable gain amplifier (VGA) with the help of the automatic gain control (AGC) method. The I\&Q low-pass filters (LPF) is used to select the preferred bandwidth to be sampled.

The effect of impulsive noise (IN) is considered for investigating the performance of the eigenvalue-based spectrum sensing scheme. Efforts are put in investigation about the influence of impulsive noise in the CR receivers in perspective of spectrum sensing.

Various models are proposed to exemplify the IN [29-33]. The model presented in [30] is used for performance evaluation in which the white noise signal is used to generate the IN waveform and shown in Fig.2.

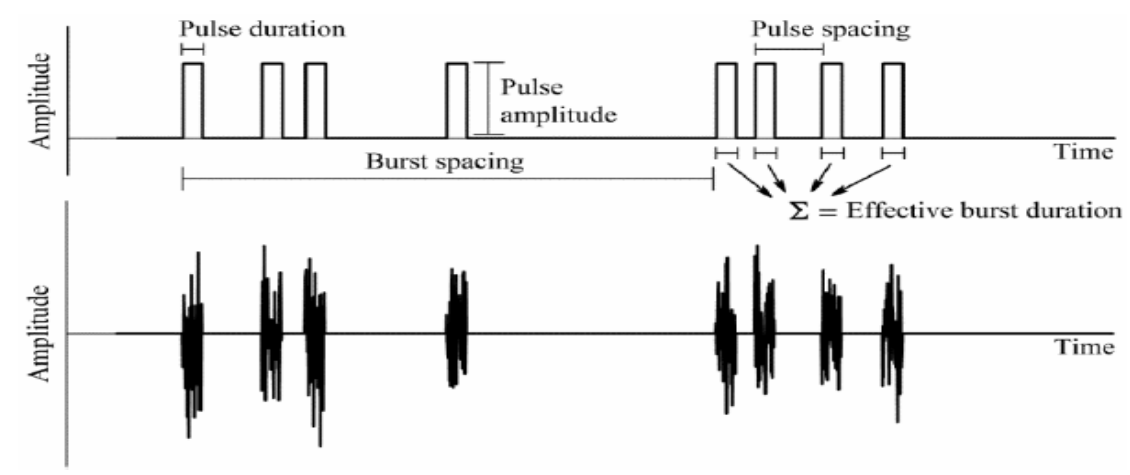

Figure 2. Impulsive Noise waveform

Considering the above mentioned issues, it is clear that the IN can cause degradation in performance of eigenvalue based spectrum sensing. Our work focus point is to assess the performance of spectrum sensing techniques by addressing the effect of IN and investigate the comparative performance in realistic implementation oriented model (R-model).The work contributes examination of the effect of impulsive noise in five different detection techniques namely GLRT, RLRT, CSFD, MMED and ED . 
The rest of the paper is organized as follows. Section 2 briefly describes the system model for spectrum detection techniques under examination. Simulation setup for performance analysis of the five spectrum sensing techniques with configuration of parameters is presented in Section 3 .Simulation results with comparative sensing performance is illustrated in Section 4. Finally conclusions are drawn in Section 5.

\section{SYSTEM MODEL}

The system model of detection is under the test of following two hypotheses $\mathrm{H}_{0}$ and $\mathrm{H}_{1}$ [34-35].

$\mathrm{H}_{0}$ : signifies the absence of the signal and presence of only noise.

$\mathrm{H}_{1}$ : signifies the presence of both signal and noise.

Thus, for the two state hypothesis numbers of cases are:-

1) Probability of Detection $\left(\mathrm{P}_{\mathrm{d}}\right)$ : i.e $\mathrm{P}\left(\mathrm{H}_{1} / \mathrm{H}_{1}\right)$, corresponds $\mathrm{H}_{1}$ to be true for the presence of primary signal.

2) Probabilty of Missed Detection $\left(\mathrm{P}_{\mathrm{md}}\right)$ : i.e $\mathrm{P}\left(\mathrm{H}_{0} / \mathrm{H}_{1}\right)$, corresponds $\mathrm{H}_{0}$ to be true for the presence of primary signal.

3) Probabilty of False Alarm $\left(\mathrm{P}_{\mathrm{fa}}\right)$ : i.e $\mathrm{P}\left(\mathrm{H}_{1} / \mathrm{H}_{0}\right)$, corresponds $\mathrm{H}_{1}$ to be true for the presence of primary signal.

Probability of detection $P_{d}$ and Probability of false alarm $P_{f a}$ can be expressed as [12],

$$
\begin{aligned}
& P_{d}=P\left(T>\gamma \mid H_{1}\right) \\
& P_{f a}=P\left(T>\gamma \mid H_{0}\right)
\end{aligned}
$$

Where, $P$ denotes the probability of a given event, $T$ is the detection-dependent test statistic and $\gamma$ is the decision threshold. The value of $\gamma$ is selected depending on the requirements for the spectrum sensing performance, which is typically evaluated through receiver operating characteristic (ROC) curves that show $\mathrm{P}_{\mathrm{fa}}$ versus $\mathrm{P}_{\mathrm{d}}$ as they vary with the decision threshold $\gamma$. For constructing test statics, the multi-sensor detection setting situation is considered, with $\mathrm{K}$ sensors (receivers or antennas) and $\mathrm{N}$ time samples. Let $\mathrm{y}(\mathrm{n})=[\mathrm{y} 1(\mathrm{n}) \ldots \mathrm{yK}(\mathrm{n})]^{\mathrm{T}}$ be the $\mathrm{K} \times 1$ received vector at time $\mathrm{n}$, where the element $\mathrm{yk}(\mathrm{n})$ is the discrete baseband complex sample at receiver $\mathrm{k}$. Under $\mathrm{H}_{0}$, the received vector consists of $\mathrm{K}$ complex Gaussian noise samples with zero mean and variance $\sigma_{\mathrm{v}}^{2}$.

$$
y(n) \mid H_{0}=v(n)
$$

Where $\mathrm{v}(\mathrm{n}) \sim \mathrm{NC}\left(0_{\mathrm{K} \times 1}, \sigma_{\mathrm{v}}^{2} \mathrm{I}_{\mathrm{K} \times \mathrm{K}}\right.$. Under $\mathrm{H}_{1}$, in contrast, the received vector contains signal plus noise,

$$
y(n) \mid H_{1}=x(n)+v(n)=h s(n)+v(n)
$$

The $s(n)$, represent the transmitted signal sample, modelled as a Gaussian2 random variable with zero mean and variance $\sigma_{s}^{2}$ and $h$ is the $K \times 1$ unknown complex channel vector. The 
assumption for the channel is made to be memory less and constant during the detection time. Under $\mathrm{H}_{1}$, the $\mathrm{SNR}$ at the receiver is defined as,

$$
\rho \cong \frac{E\|x(n)\|^{2}}{E\|v(n)\|^{2}}=\frac{\sigma_{S}^{2}\|h\|^{2}}{\sigma_{v}^{2} K}
$$

where, $\|$.$\| denotes Euclidean (L2) norm.$

The received samples are stored by the detector in the $\mathrm{K} \times \mathrm{N}$ matrix

$$
\mathrm{Y} \cong,[\mathrm{y}(1) \ldots \mathrm{y}(\mathrm{N})]=\mathrm{hs}+\mathrm{V}
$$

Where, $\mathrm{s} \cong,[\mathrm{s}(1) \ldots \mathrm{s}(\mathrm{N})]$, is a $1 \times \mathrm{N}$ signal vector and $\mathrm{V} \cong,[\mathrm{v}(1) \ldots \mathrm{v}(\mathrm{N})]$ is a $\mathrm{K} \times \mathrm{N}$ noise matrix. The sample covariance matrix $\mathrm{R}$ is then defined as,

$$
\mathrm{R} \cong \frac{1}{\mathrm{~N}} \mathrm{YY}^{\mathrm{H}}
$$

Let $\lambda_{1} \geq \cdots \geq \lambda_{\mathrm{K}}$ be the eigen values of $\mathrm{R}$ (without loss of generality, sorted in decreasing order).

We focus on the difference in detection performance between the cases of known and unknown noise level.

The test statistics for GLRT, CSFD, MMED, ED and RLRT are respectively calculated according to [25].

$$
\begin{gathered}
\mathrm{T}_{\mathrm{GLRT}}=\frac{\lambda_{1}}{\frac{1}{\mathrm{~m}} \operatorname{tr}(\mathrm{R})}=\frac{\lambda_{1}}{\frac{1}{\mathrm{~m}} \sum_{\mathrm{i}=1}^{\mathrm{m}} \lambda_{\mathrm{i}}} \\
\mathrm{T}_{\mathrm{CSFD}}=\frac{\lambda_{1}}{\lambda_{\mathrm{m}}} \\
\mathrm{T}_{\mathrm{MMED}}=\frac{\lambda_{1}}{\sigma^{2}} \\
\mathrm{~T}_{\mathrm{ED}}=\frac{\|\mathrm{Y}\|_{\mathrm{F}}^{2}}{\mathrm{mn} \sigma^{2}}=\frac{1}{\mathrm{~m} \sigma^{2}} \sum_{\mathrm{i}=1}^{\mathrm{m}} \lambda_{\mathrm{i}}, \\
\mathrm{T}_{\mathrm{RLRT}}=\frac{\lambda_{1}}{\sigma_{\mathrm{v}}^{2}}
\end{gathered}
$$

Where, $\sigma^{2}$ is the thermal noise power, expected to be known and with equal value in each sensor input, and $\operatorname{tr}()$ and \|\|$_{F}$ are the trace and the Frobenius norm of the underlying matrix, respectively.

\section{Simulation SeTUP}

With reference to receiver architecture shown in the Fig.1, the simulation setup is build and the, performance parameters configured are as follows, 
- $\mathrm{m}$ : Antennas in CR / CR with one antenna each.

- $n$ : Number of received samples collected from primary transmitter.

- Ne: The number of Monte Carlo simulation events.

-The sensing techniques under analysis are ED, MFD, CSFD, GLRT and RLRT.

- The type of transmitted signal (noise, BPSK, QAM or user defined modulation).

- $\mathrm{N}_{\mathrm{q}}$ : Number of quantization levels .

- D: ADC dynamic range.

- $\mathrm{f}_{\mathrm{od}}$ : overdrive factor.

For impulsive noise (IN) following additional parameters have to be set,

- $\mathrm{p}_{\mathrm{IN}}$ : Probability of IN occurrence.

- $\mathrm{p}_{\mathrm{CR}}$ : Fractions of CR hits by IN.

- $\mathrm{N}_{\mathrm{S}}$ : Number of IN blurts.

- $\mathrm{K}$ : The ratio between average IN power and average thermal noise power.

- $\beta$ : The average number of samples between impulsive noise pulses.

- A : The mean of the log-normal impulsive noise amplitudes.

- B : The standard deviation of the log-normal amplitudes.

Two different simulation processes are carried out. For the first simulation type, the SNR is kept at fixed value in $\mathrm{dB}$ and the decision threshold range is varied between minimum and maximum threshold values. While the second process of simulation is carried out by defining three parameters, like the fixed threshold level, and the minimum and the maximum SNR values in $\mathrm{dB}$ with the number of points within the SNR range.

\section{Simulation RESUlts}

Two major parameters used as a performance measurement metrics to analyse the performance of detection process are, Probability of Detection $\left(\mathrm{P}_{\mathrm{d}}\right)$ and Probability of False alarm $\left(\mathrm{P}_{\mathrm{fa}}\right)$. The performance of a spectrum sensing techniques is illustrated by the receiver operating characteristics (ROC) curve which is a plot of $P_{d}$ versus $P_{f a}$.

The performance measurements parameters set for simulation type one as, $m=8, n=50$, SNR $=-10 \mathrm{~dB}$, no. of monte carlo events simulated $=1000$ and minimum to maximum threshold levels set in the range from $\gamma=0.78$ to $\gamma=1.1$ with 8 different threshold events. In the second simulation scenario the threshold level is kept fixed at value $\gamma=1.4$ and SNR is varied in between the range of $-10 \mathrm{~dB}$ to $20 \mathrm{~dB}$. 


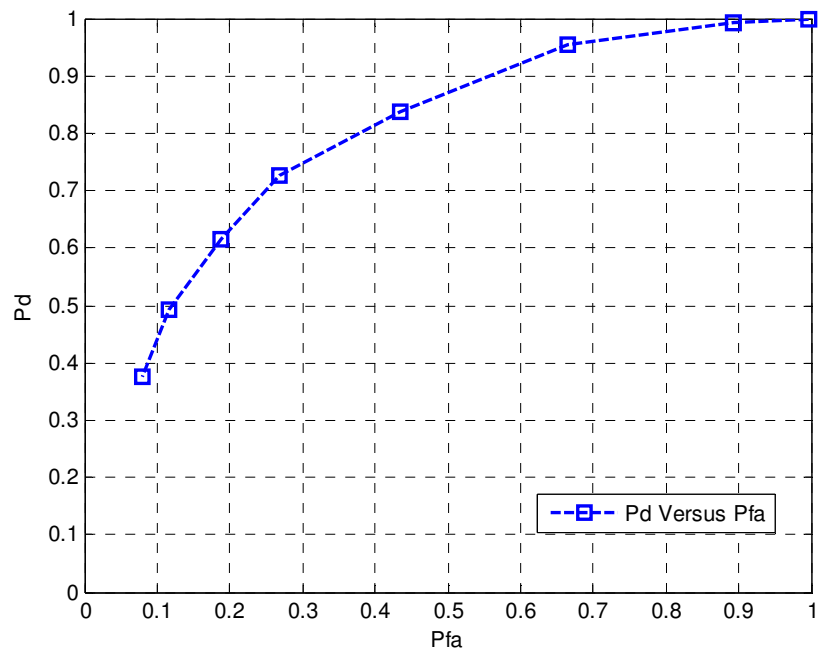

Figure 3. ROC curve with $\mathrm{SNR}=10 \mathrm{~dB}$ for $\mathrm{m}=8$ and $\mathrm{n}=50$

Fig. 3, represents the ROC curve for ED method, it is evident from the graph that for less $\mathrm{P}_{\mathrm{fa}}$ values, $P_{d}$ is comparatively high. But, as $P_{f a}$ increased, there is increased significant improvement is observed in $P_{d}$. Thus, it can be seen that the detection performance is improved in ED at low SNR values.

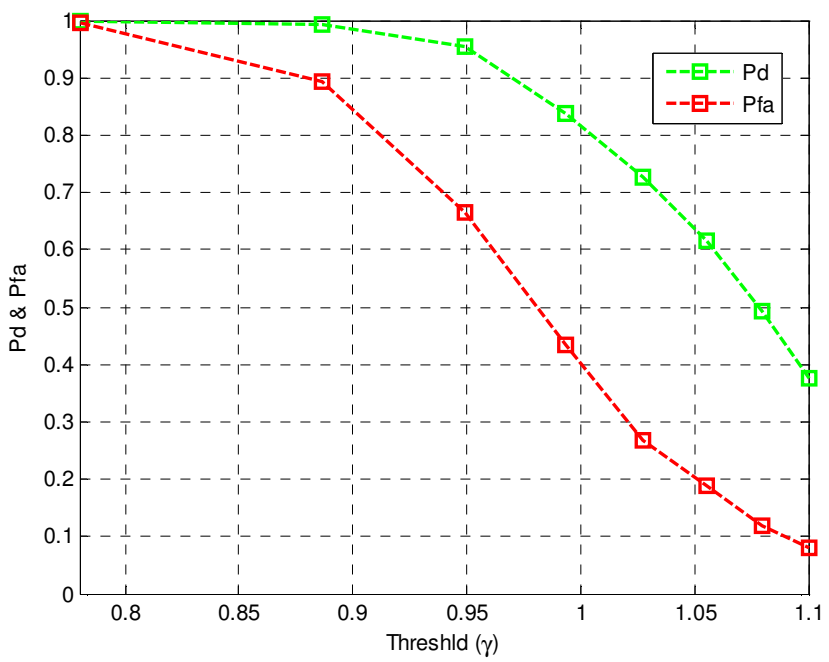

Figure 4. Pd, Pfa Vs Threshold

Fig.4. presents comparison of $\mathrm{P}_{\mathrm{d}} \mathrm{P}_{\mathrm{fa}}$, with respect to change in threshold levels. Fixed a given threshold, the behaviour of the $\mathrm{P}_{\mathrm{fa}}$ is shown, it is evident from the results that the $\mathrm{P}_{\mathrm{d}}$ is improved. 
International Journal of Wireless \& Mobile Networks (IJWMN) Vol. 6, No. 6, December 2014

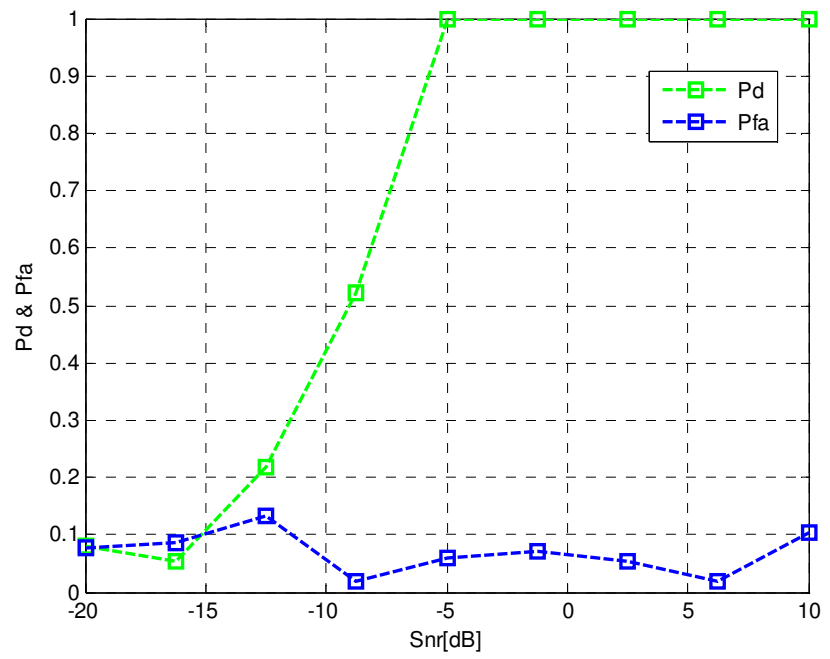

Figure 5. Pd, Pfa Vs SNR

In Fig.5 the relation between the $\mathrm{P}_{\mathrm{d}}, \mathrm{P}_{\mathrm{fa}}$ with SNR is verified. With increase in SNR the value of $\mathrm{P}_{\mathrm{d}}$ is also increased. Also as the value of $\mathrm{P}_{\mathrm{fa}}$ vary there is significant improvement in $\mathrm{P}_{\mathrm{d}}$ is obtained. Although the results presented in Figure 3-5 are only for ED method, but we retried that this is valid for all detection techniques considered here.

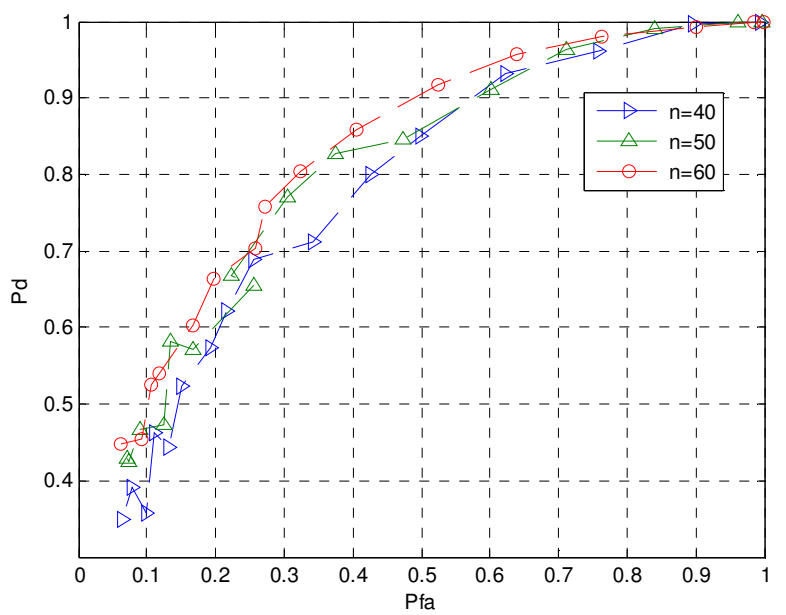

Figure 6. ROC curve for ED method with $\mathrm{m}=8$ and $\mathrm{SNR}=10 \mathrm{~dB}$ 
International Journal of Wireless \& Mobile Networks (IJWMN) Vol. 6, No. 6, December 2014

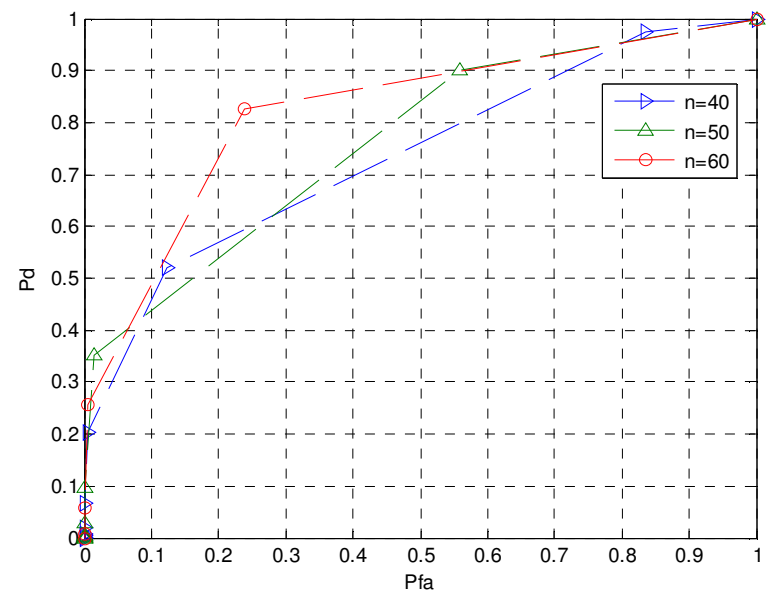

Figure 7. ROC curve for GLRT method with $\mathrm{m}=8$ and $\mathrm{SNR}=10 \mathrm{~dB}$

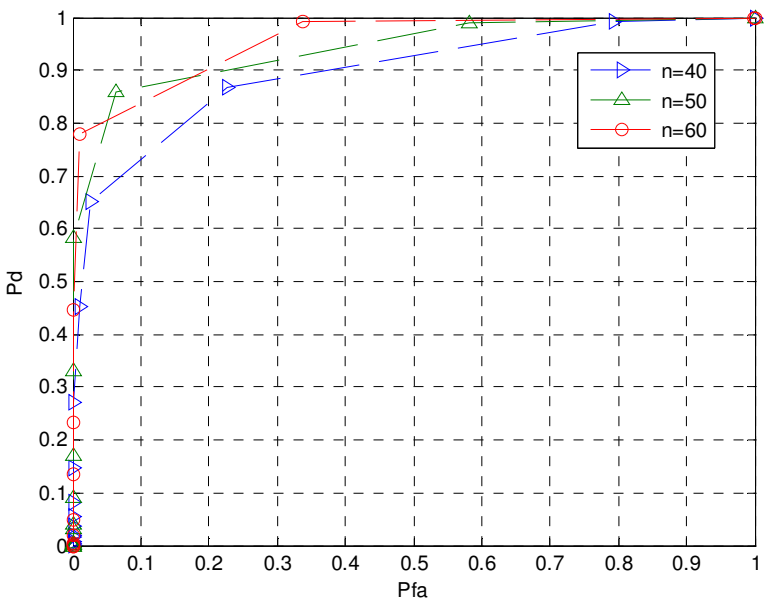

Figure 8. ROC curve for CSFD method with $\mathrm{m}=8$ and $\mathrm{SNR}=10 \mathrm{~dB}$

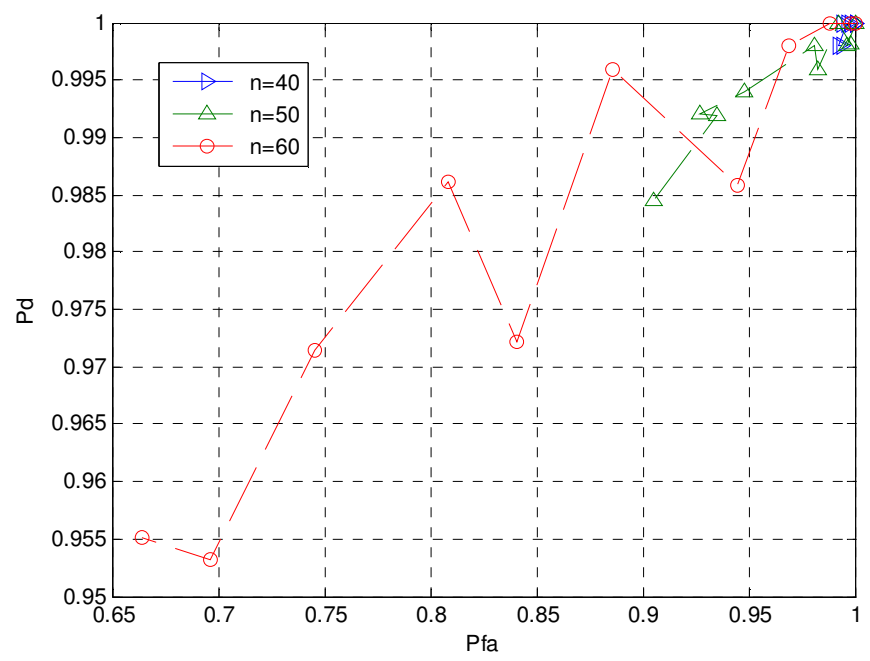

Figure 9. ROC curve for MMED method with $\mathrm{m}=8$ and $\mathrm{SNR}=10 \mathrm{~dB}$ 


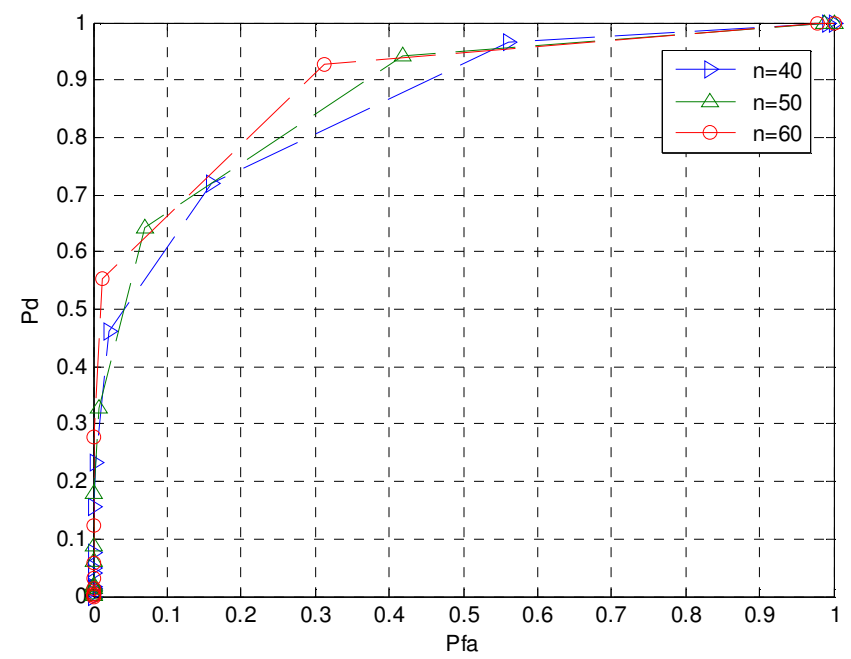

Figure 10. ROC curve for RLRT method with $\mathrm{m}=8$ and $\mathrm{SNR}=10 \mathrm{~dB}$

Fig. 6-10, shows ROC curve with effect of IN for all the detection methods under test. Different values of the number of collected samples $(n)$, concerning the probability of false alarm $\left(\mathrm{P}_{\mathrm{fa}}\right)$ and the probability of detection $\left(\mathrm{P}_{\mathrm{d}}\right)$ are measured. The results illustrate system performance under IN conditions. With reference to the number of collected samples (n), we set different minimum to maximum threshold levels as follows. For $\mathrm{n}=40$ the threshold levels are $\gamma_{\min }=$ 2.8 and $\gamma_{\max }=6.5$, for $\mathrm{n}=50, \gamma_{\min }=2.8$ and $\gamma_{\max }=6.5$, and for $\mathrm{n}=60 \gamma_{\min }=2$ to $\gamma_{\max }=4.5$. Also $\mathrm{m}=8$ and SNR is kept at $-10 \mathrm{~dB}$. The number of primary transmitters $\mathrm{p}$ $=1, \mathrm{~K}=0, \mathrm{~L}=1-20, \mathrm{D}=2, \mathrm{f}_{\mathrm{od}}=1-2$ and $\mathrm{N}_{\mathrm{q}}$ is varied as 4,8 and 256. It is clear from the results that with reference to greater threshold value, the values of $\mathrm{P}_{\mathrm{d}}$ and $\mathrm{P}_{\mathrm{fa}}$ are smaller. Similarly, for smaller threshold, $\mathrm{P}_{\mathrm{d}}$ and $\mathrm{P}_{\mathrm{fa}}$ tends to 1 . Increase in sensing performance is observed with increase in $\mathrm{n}$. It can be further confirmed from ROC curves that, the influence of increasing the number $\mathrm{n}$ of collected samples per $\mathrm{CR}$ is a performance improvement, considering as fixed the remaining system are identified in the graph.

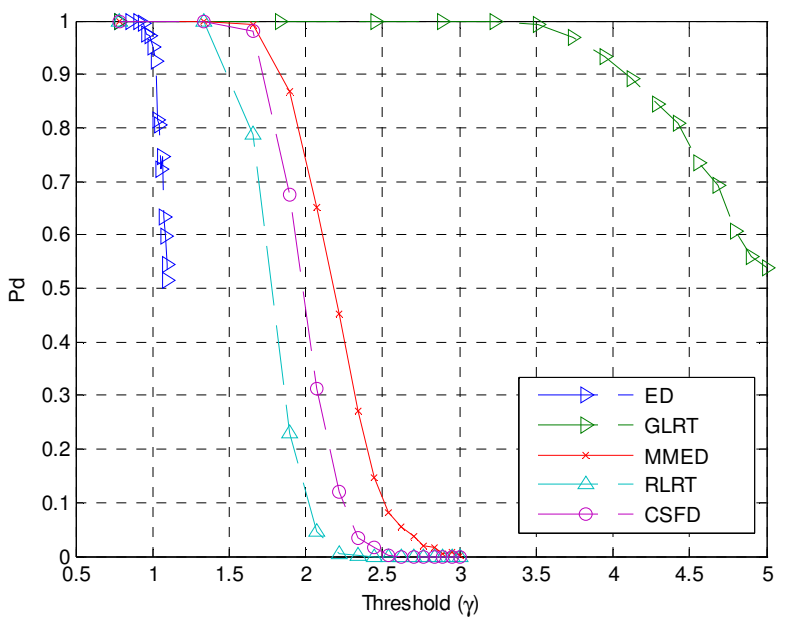

Figure 11. Pd Vs Threshold for all sensing methods 


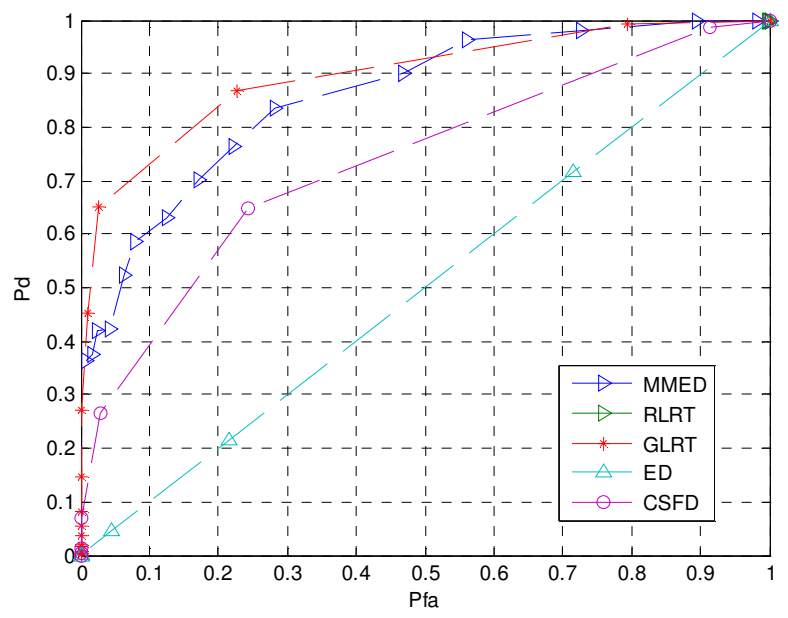

Figure 12. ROC Curve for all sensing methods under parameter variations

Fig. 11-12, illustrates the performance comparison of all the detection methods under test namely, GLRT, CSFD, MMED, ED and RLRT. Different threshold values from $\gamma_{\min }=0.5$ and $\gamma_{\max }=5$ are used for plotting the probability of detection Pd in Fig.11. From the comparative plot, it is clear that the GLRT method demonstrate better detection performance than the other detection methods for variable threshold level with fixed SNR value. Fig.12 presents ROC curves relating $\left(\mathrm{P}_{\mathrm{fa}}\right)$ and the $\left(\mathrm{P}_{\mathrm{d}}\right)$ for the investigated detection techniques, for $\mathrm{p}=1, \mathrm{~m}=6, \mathrm{SNR}=-10 \mathrm{~dB}$, and variable $N_{q}=8$, and for $f_{o d}$ and $L$ ranging from 1 to 2 , and 1 to 20, respectively. And analysis of the results indicates that, the sensing performance is improved in GLRT and MMED methods for realistic implementation oriented model in the presence of impulsive noise.

\section{Conclusions}

In this paper, performance of five different detection methods for spectrum sensing in cognitive radio networks is evaluated by implementing the realistic model with typical signal processing is involved in implementation. Closed form expressions for probability of detection and false alarm over different sensing methods are evaluated. Simulation results shows that, the effect of

impulsive noise is less in GLRT method as compare to the other detection methods and thus the performance of GLRT method is improved in realistic implementation model.

In comparison with the conventional model the realistic implementation oriented model provide reasonably better results. Moreover the comparison of results helps for performance evaluation and of sensing methods in cognitive radio networks. It is believed that the work carried out in this paper is useful to understand the performance in cognitive radio network for selection of best sensing method. In future work attention can be given on the computation of closed form analysis by using more realistic approach with typical CR signal processing tasks.

\section{ACKNOWLEDGEMENTS}

The authors would like to thank everyone, just everyone! 
International Journal of Wireless \& Mobile Networks (IJWMN) Vol. 6, No. 6, December 2014

\section{REFERENCES}

[1] Federal Communications Commission, "Spectrum policy task force report," ET Docket No. 02-135; November 2002.

[2] J.Mitola , Maguire G.Q.," Cognitive Radio: making software radios more personal,” IEEE Personal Commu.Mag., 1999;6:13-8.

[3] J. Mitola, III, "Cognitive radio for flexible mobile multimedia communications," in Proc. IEEE Mobile Multimedia Commun. Conf., San Diego, CA, Nov. 1999, pp. 3-10.

[4] I.F.Akyildiz., “ Cooperative spectrum sensing in cognitive radio networks: A survey: a survey,"In: Elsevier physical communication,2011,pp.40-62.

[5] A. Sahai and D. Cabric, "Spectrum sensing: Fundamental limits and practical challenges," In proc. IEEE Int.Symp.New Fronties DySPAN (Tutorial),Baltimore,MD Nov.2005.

[6] S. M. Kay, "Fundamentals of Statistical Signal Processing: Detection Theory," Upper Saddle River, NJ: Prentice-Hall, 1998.

[7] H. S. Chen, W. Gao, and D. G. Daut, "Signature based spectrum sensing algorithms for IEEE 802.22 WRAN," in Proc. IEEE Int. Conf. Commun., Glasgow, U.K., Jun. 24-28, 2007, pp. 6487-6492.

[8] D. Cabric, A. Tkachenko, and R. W. Brodersen, "Spectrum sensing measurements of pilot, energy, and collaborative detection," in Proc. MILCOM, Washington, DC, Oct. 23-25, 2006, pp. 1-7.

[9] H Urkowitz, "ED of unknown deterministic signals," Proc. IEEE, vol. 55, pp. 523-531, Apr. 1967.

[10] S. Atapattu, C. Tellambura and H.Jiang, "Analysis of Area under ROC curve of energy detection" IEEE Transactions On Wireless Communications,Vol.9,No.3, pp. 1216-1225, 2010.

[11] V. I. Kostylev, "Energy detection of a signal with random amplitude," in Proc. IEEE ICC, Apr. 2002, vol. 3, pp. 1606-1610.

[12] F.F.Digham, M.S. Alouiniand M.K. Simon,“ Energy detection of unknown signals over fading channels”, IEEE Transactions on Communications,Vol.5,No.1,pp.21- 24,2007.

[13] W. A. Gardner, "Signal interception: A unifying theoretical framework for feature detection," IEEE Transactions on Communications, vol. 36, no. 8, pp. 897-906, Aug. 1988.

[14] W. Gardner, "A new method of channel identification," IEEE Transactions on Communications, vol. 39, no. 6, pp. 813-817, Jun. 1991.

[15] P. D. Sutton, K. E. Nolan, and L. E. Doyle, "Cyclostationary signatures in practical cognitive radio applications,” IEEE J. Sel. Areas Commun. vol. 26, no. 1, pp. 13-24, Jan. 2008.

[16] D. Cabric, S. M. Mishra, and R. W. Brodersen, "Implementation issues in spectrum sensing for cognitive radios," in Proc. 38th Asilomar Conf. Syst. Comput., Pacific Grove, CA, Nov. 7-11, 2004, pp. 772-776.

[17] Y.Zeng , Y-C Liang, “ Eigen value-based spectrum sensing algorithms for cognitive radioCommunications," IEEE Transactions on communications, 2009;57(6):1784-93. 2005.

[18] B.Nadler,F. and R. Garello, "Performance of Eigenvalue-Based Signal Detectors with Known and Unknown Noise Level ," In procedddings of IEEE International Conference on communications,ICC,Kyto,Japan,5-9 June 2011;pp.1-5.

[19] A. Kortun,T. Ratnarajah,M. Sellathurai,C.Zhong and C.Papadias, "On the performance ofeigenvaluebased cooperative spectrum sensing for cognitive radio", IEEE Journal on Sel. Top. Signal Process. 2011, 5, 49-55.

[20] R. Tandra and A. Sahai, "Fundamental limits on detection in low SNR under noise uncertainty," in Proc. Int. Conf. Wireless Netw., Commun.Mobile Comput., Maui, HI, Jun. 13-16, 2005, pp. 464 469.

[21]M-R-Osorio,de Haro-Ariet, L Calvo-Ramon, L.M.; Sanchez, M.G., "Performance evaluation of WCDMA in actual impulsive noise scenarios using adaptive antennas",IEEE Proc. Commun. 2004, 151, 589-594.

[22] M..Budsabathon and S.Hara,"Robustness of OFDM Signal Against Temporally Localized Impulsive Noise", In Proceedings of the IEEE VTS 54th Vehicular Technology Conference, VTC 2001 Fall, Atlantic City, NJ, USA, 7-11 October 2001; Volume 3, pp. 1672-1676.

[23] D.P.Patil,V.M.Wadhai, "Performance Evaluation of Spectrum Sensing in Cognitive Radio for Conventional Discrete-time Memory less MIMO Fading Channel Model," In Procedding of The 10th International Conference on Collaborative Computing: Networking, Applications and Worksharing (CollaborateCom2014) Miami, FL, USA, October 22-25, 2014. 
International Journal of Wireless \& Mobile Networks (IJWMN) Vol. 6, No. 6, December 2014

[24] D.A.Guimarães, R.A.de Souza, andA.A. dos Anjos, "Implementation orientation model for centralized data fusion cooperative spectrum," IEEE Communations letter ,2012,16,184-187.

[25] D.A.Guimarães, R.A.de Souza, andA.A. dos Anjos, "Simulation Platform for Performance Analysis of Cooperative Eigenvalue Spectrum Sensing with a Realistic Receiver Model Under Impulsive Noise," Vehicular Technologies-Deployment and Applications, 2013.

[26] B. Razavi, "Design considerations for direct-conversion receivers," IEEE Trans. Circuits Syst. II, vol. 44, no. 6, , June 1997,pp. 428-435.

[27] R.Svitek and S,Raman,"DC offsets in direct-conversion receivers: characterizationand implications,”IEEE Microwave Magzene,Vol.6,no.3,pp.76-78,September 2005.

[28] M. Keshavarzi, A. Mohammadi, and A. Abdipour, "Characterization and compensation of DC offset on adaptive MIMO direct conversion transceivers," IEICE Trans. Commun., vol. E94-B, no. 1, pp. 253-261, Jan. 2011.

[29] I.Mann ,S. McLaughlin,W. Henkel,R. Kirkby and T.Kessler, “ Impulse generation with appropriate amplitude, length, inter-arrival, and spectral characteristics," IEEE Journal on Selected Areas in Communications,. 2002;20(5):901-12.

[30] J.Lago- Fernández and J.Salter, "Modelling Impulsive Interference in DVB-T: Statistical Analysis, Test Waveforms and Receiver Performance," BBC R\&D white paper WHP 080, Apr. 2004.

[31] D.Middleton, "Non-Gaussian noise models in signal processing for telecommunications: new methods an results for class A and class B noise models," Information Theory, IEEE Transactions on. 1999;45(4):1129-49.

[32] P.Torio and Sanchez MG., " Generating Impulsive Noise," [Wireless Corner]. Antennas and Propagation Magazine, IEEE. 2010;52(4):168-73.

[33] P. Torio and Sanchez MG, Cuinas I., "An algorithm to simulate impulsive noise. Software, Telecommunications and Computer Networks, “(SoftCOM), 2011 19th International Conference on. 2011. p. 1-4.

[34] M.Srinath,P.Rajasekaran, and R.Vishwanathan, "Introduction to stactialsignal processing and appilcations,"Prientice Hall,Eaglewood Cliffs,07692,1996.

[35] R.D.Yates and D. Goodman, "Probability and Stochastic Processes: A Friendly Introduction for Electrical and Computer Engineers." 2nd ed. 2004.

\section{Authors}

Dipak P.Patil Received his Bachelours degree in Engineerng in the field of Electronics and Telecommunications in the year 2000 from North Maharashtra University,Jalgaon and Masters from SGSITS Indore in the year 2007. He is cureently doing his research work at the department of Applied Electronics, at Sant Gadgebaba Amravati University,Amravati.His area of research is Wireless Communication and Cognitive Radio.

.Vijay M.Wadhai received his Ph.D. degree in Electronics Engineering from Santadgebaba Amravati University, Amravati in 2007. Provides technical guidance to working professional in Industry and Academics for research work.Recognised $\mathrm{PhD}$ supervisor in Computer Enggineering and Electronics \& Telecommunication Enggineering .Guiding PhD / Reserach students in cognitive radio, wireless network, VANET, Body Area Network

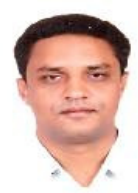
domain.

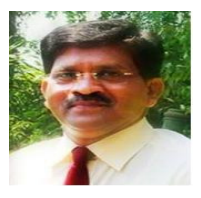

